\title{
FunZioni IdENTITARIE dell' Alternanza LINGUISTICA IN APPRENDENTI DI ITALIANO DURANTE LO SVOLGIMENTO DI Attività in Gruppo
}

\author{
Roberta Ferron ${ }^{*}$
}

ABSTRACT: Questo articolo indaga, servendosi degli strumenti teorici messi a disposizione dall'analisi della conversazione, coadiuvata da una serie di nozioni e riferimenti tratti dagli studi che esaminano la struttura dell'interazione nella classe di lingua, i diversi usi che gli apprendenti di italiano LS fanno delle lingue presenti nella classe, specialmente della loro LM, durante lo svolgimento di attività di gruppo per dare voce alle multiple identità che costituiscono ciascun individuo. Dallo studio emerge che l'identità degli apprendenti è in continua trasformazione. Infatti, per potersi affermare, gli apprendenti dovranno "interpretare" un insieme di ruoli che cambiano e che dovranno negoziare in base al contesto d'interazione.

PAROLE CHIAVE: interazione fra pari; lingue affini; analisi della conversazione; alternanza di codice; identità.

RESUMO: Este artigo, valendo-se dos instrumentos teóricos elaborados pela análise da conversação, além de uma série de noções e referências extraídas de trabalhos que examinam a estrutura da interação na sala de aula de língua, reflete sobre os usos diferentes que os estudantes de italiano LE fazem das línguas presentes, especialmente da LM, durante o desenvolvimento de atividades em grupo, para expressar as variadas identidades que constituem cada indivíduo. Do estudo

* Docente de Língua Italiana na Universidade de São Paulo (USP). 
aflora que a identidade dos estudantes está em contínua transformação. Com efeito, para conseguir se afirmar, os estudantes deverão "interpretar" um conjunto de papéis, que mudam e que eles deverão negociar com base no contexto da interação. PALAVRAS-CHAVE: interação entre pares; línguas afins; análise da conversação; alternância de código; identidade.

ABSTRACT: This article, taking advantage of the theoretical tools developed by conversation analysis, plus a series of notions and references extracted from works that examine the structure of the interaction in the language classroom, reflects on the different uses that LE Italian students make of the languages present in class, especially the LM, during the development of group activities, to express the various identities that constitute each individual. The study suggests that the identity of the students are in continuous transformation. Indeed, to be able to affirm themselves, students must "interpret" a set of roles that change and that they should negotiate based on the context of the interaction.

KEYWORDS: interaction between pairs; related languages; conversation analysis; code switching; identity. 


\section{Introduzione}

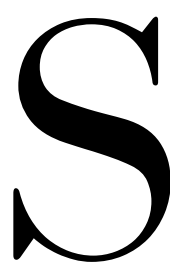

ebbene per molto tempo gli studi relativi all'acquisizione/apprendimento della lingua straniera (d'ora in poi LS) abbiano privilegiato approcci di stampo cognitivista (si vedano a questo proposito CLARK; CLARK, 1977; STERN, 1983), da alcuni anni a questa parte, grazie anche all'avvento dell'analisi della conversazione (d'ora in poi AC), che ha profondamente destabilizzato le concezioni tradizionali secondo le quali la lingua sarebbe principalmente un sistema di forme e l'acquisizione un processo puramente cognitivo messo in atto dall'apprendente per il raggiungimento di una certa competenza linguistica nella lingua target (cfr. FIRTH e WAGNER, 1997; PEKAREK, 2006, 2010), è sorto un certo interesse anche per lo studio dei legami che intercorrono tra l'apprendimento della LS e l'identità degli apprendenti. In questo senso, sia le ricerche svolte in ambito post-strutturalista, come, per esempio, quelle di Norton Peirce (1995), Norton (1997, 2000), Pavlenko (2001), Pavlenko e Blackledge (2004), Kramsch 
(2007), Block (2007) e Ellwood (2008, 2011), sia le indagini condotte in Brasile e ispirate all'analisi del discorso di stampo francese (si vedano, fra gli altri, SERRANI-INFANTE, 1997; CELADA, 2008), dalle quali emerge che il contatto con la LS promuove una risignificazione della formazione identitaria del soggetto, devono essere interpretate come degli ulteriori passi in avanti per approfondire una tematica così complessa come quella dell'identità e l'apprendimento della LS.

Le indagini di stampo post-strutturalista - si vedano, fra gli altri, Norton Peirce (1995), Norton (1997), Pavlenko (2001), Liddicoat e Crozet (2001), Lantolf e Genung (2002), Pavlenko e Blackledge (2004), Block (2007), Kramsch (2007) - tendono a evidenziare che l'identità è una costruzione di natura flessibile e non stabile e che non dipende semplicemenle dall' appartenenza ad un gruppo sociale, culturale o linguistico, ma, in contesti educativi, è cocostruita, negoziata e soggetta ad una continua trasformazione per mezzo della lingua. Da queste ricerche affiora che nella classe di LS sono effettivamente poche le opportunità che permettono agli apprendenti di far emergere la loro identità sia perché agli studenti è richiesta una competenza linguistica e concettuale che non possiedono in LS sia perché la tendenza dell'insegnante è quella di esercitare un certo potere sugli studenti.

Tuttavia, nonostante i progressi teorici, rimangono ancora molte zone d'ombra su cui far luce. Ad esempio gli studi citati sono stati condotti, in gran parte, fra apprendenti la cui lingua materna (d'ora in poi LM) è tipologicamente molto distante dalla LS (cfr. NORTON PEIRCE, 1995; NORTON, 1997, 2000; LIDDICOAT e CROZET, 2001; LANTOLF e GENUNG, 2002; PAVLENKO; BLACKLEDGE, 2004; BLOCK, 2007; ELLWOOD, 2008, 2011), mentre i pochi lavori dedicati ai ruoli identitari che i discenti tendono ad assumere quando stanno apprendendo una lingua affine alla propria LM, non sono particolarmente interessati a descrivere il contesto in cui ha luogo l'interazione (cfr. SERRANI-INFANTE, 1997; CELADA, 2008).

Inoltre, una fra le metodologie più utilizzate per lo studio dell'identità consiste nella raccolta e nella successiva analisi di racconti autobiofgrafici, come ad esempio, monologhi, interviste spontanee o guidate (cfr. NORTON PEIRCE, 1995; NORTON, 2000; PAVLENKO, 2001; JOHNSON e GOLOMBEK, 2002; BLOCK, 2007; NUNAN e CHOI, 2010). Ora, sebbene questa modalità consenta di dar voce al proprio parlato intimo, è pur vero che tralascia tutti gli aspetti legati all'interazione fra i discenti, fattore che non può essere sottovalutato, visto che, secondo una prospettiva dinamica, è per mezzo dell'interazione che le identità vengono negoziate.

Il presente contributo prende spunto da uno studio condotto da Ellwood (2008) in cui, analizzando i dati raccolti in una classe di lingua inglese (L2), composta da studenti con un 
background linguistico variegato, la ricercatrice illustra come il passaggio alla propria $\mathrm{LM}^{1}$ possa offrire agli studenti la possibilità di esprimere ciò che effettivamente sono. Per farlo ricorrono a degli "atti di identità", espressione coniata da Le Page e Tabouret-Keller (1985), per indicare gli atti linguistici attraverso i quali i parlanti rivelano sia la propria identità personale, sia il ruolo sociale al quale aspirano. Nel corso della sua ricerca Ellwood (2008) distingue tre atti che definisce: atti di allineamento, atti di resistenza e atti di identità globale. I primi due sono realizzati attraverso il passaggio alla LM, mentre gli atti di identità globale sono espressi con la LM del compagno ${ }^{2}$.

Fatta questa premessa i dati che presentiamo, raccolti presso un'università brasiliana dello Stato di San Paolo del Brasile, serviranno per analizzare i diversi usi che apprendenti di italiano LS fanno delle lingue presenti nella classe, specialmente della loro LM, ovvero il portoghese brasiliano, durante lo svolgimento di attività di gruppo per dare voce alle multiple identità che costituiscono ciascun individuo.

Attraverso il seguente contributo, ci proponiamo di verificare:

- a quale tipologia di atti ricorrono studenti che condividono la stessa LM;

- che caratteristiche linguistiche assumono gli atti di identità in un contesto caratterizzato da lingue fra loro affini.

L'analisi è condotta a partire dall' AC, coadiuvata da una serie di nozioni e riferimenti tratti dagli studi che esaminano la struttura dell'interazione nella classe, specie in quella di lingua (ORLETTI, 2000; NUSSBAUM; UNAMUNO, 2000; FELE; PAOLETTI, 2003; CAMBRA, $2003,2005)$. Questo tipo di approccio ha infatti il vantaggio di mettere in risalto la dinamica interattiva e tutte le procedure messe in atto dai parlanti a livello locale.

Dato che quando si parla di identità, ci si riferisce ad un costrutto estremamente complesso, nel presente lavoro facciamo riferimento alle teorie che, nate sotto l'impulso del costruttivismo

1 In questo contesto, useremo indistintamente alternanza di codice o cambio di codice, per indicare l'uso alternato di due lingue dentro lo stesso turno o dentro lo stesso enunciato. L'alternanza di codice è fondamentale ai fini dell'acquisizione/apprendimento della LS perché consente agli apprendenti di assicurare la gestione globale delle loro possibilità comunicative (PY, 1994).

2 Ellwood (2008) definisce atti di allineamento (Acts of Classroom Alignment) tutti quei commenti in cui gli studenti, nonostante dimostrino delle difficoltà nello svolgimento del compito, per non perdere la faccia e per rivendicare il ruolo di "bravi studenti", dissimulano una serie di interventi in cui la LM serve per dimostrare un certo sforzo e impegno al fine di compiacere l'insegnante. In questo caso il cambio di codice servirà per porre maggiore attenzione ai fini dello svolgimento del compito. Sono atti di resistenza (Acts of Classroom Resistance), quelli in cui gli apprendenti si rifiutano di accettare il ruolo di studenti e, attraverso commenti espressi in LM, dichiarano una certa ostilità o sull'attività che stanno svolgendo, o nei confronti dell'insegnante. In questo caso il cambio di codice, oltre ad offrire la possibilità di dar voce alla propria identità, permette all'apprendente di esprimere una difficoltà a proposito dell'attività in corso, fornendo all'insegnante alcune utili indicazioni didattiche. Attraverso gli atti di identità globale (Acts of Global Identity), gli studenti usano, a volte in modo scorretto, la lingua del compagno per soddisfare il desiderio di mostrare una identità cosmopolita. 
sociale, tendono a considerare l'identità come un'entità dinamica e flessibile, costituita più che da un insieme fisso di tratti, da un repertorio di possibili "ruoli" identitari (si vedano, ad esempio, RAMPTON, 1995; ANTAKI e WIDDICOMBE, 1998; DE FINA, SCHIFFRIN e BAMBERG, 2006; DE FINA, 2007; CILIBERTI, 2007).

Questo approccio, diventato dominante tra gli studiosi di sociolinguistica, ha l'enorme vantaggio di basarsi sull'osservazione minuziosa dei comportamenti sociali e linguistici in un contesto determinato. Ogni individuo, pertanto, non avrebbe un'unica identità, ma piuttosto diverse identità potenziali, all'interno delle quali può spostarsi o essere spostato a seconda del contesto in cui si trova ad agire e comunicare.

\section{Il contesto della ricerca}

I dati che esaminiamo nel corso del presente contributo, sono stati raccolti in un contesto universitario. La classe di livello A2/B1 (Consiglio d'Europa, Quadro Comune Europeo di Riferimento - QCER, 2002), affidata ad un insegnante di madrelingua italiana formatosi in Italia, era composta da sette apprendenti di italiano LS, tutti aventi come LM il portoghesebrasiliano. Siccome il contesto viene definito anche a seconda delle attività che i partecipanti stanno svolgendo (GAJO, KOCH e MONDADA, 1996), è bene precisare che gli studenti erano intenti a svolgere dei task, attività che "[...] coinvolgono gli apprendenti nella comprensione, manipolazione, produzione o interazione nella lingua target in quanto l'attenzione è focalizzata principalmente sul significato, piuttosto che sulla forma" (NUNAN, 1989, p. 10, traduzione nostra), la cui finalità era quella di promuovere l'interazione e la negoziazione. La tipologia delle attività era piuttosto varia anche se per questo studio sono state prese in considerazione le attività svolte in piccoli gruppi, volte a favorire l'uso della lingua orale.

\section{Metodologia}

La ricerca, che si è avvalsa degli strumenti messi a disposizione dall'approccio etnografico (VAN LIER, 1988; WATSON-GEGEO, 1997) ed è stata basata sull'osservazione di un gruppo classe. Il corpus è composto da un totale di 15 ore di registrazione audio.

Durante le registrazioni o immediatamente dopo sono stati presi alcuni appunti sintetici, comprendenti uno schema del setting con la disposizione dei posti e dei nomi degli allievi e 
degli insegnanti e alcune annotazioni sull' argomento della lezione. La modalità di osservazione che abbiamo adottato è quella a metà strada tra i due poli che Duranti $(1997$, p. 99) definisce "partecipazione passiva" e "partecipazione completa", visto che siamo intervenuti tutte le volte in cui i partecipanti ci invitavano a prendere parte agli eventi comunicativi. Durante la fase di trascrizione, abbiamo a volte avuto difficoltà nel comprendere quanto detto dagli studenti nel corso delle interazioni, che potevano risultare incomprensibili a causa dei rumori, delle sovrapposizioni di voci e perché parlavano a voce molto bassa. Per questo motivo le sequenze che prendiamo in esame possono, a volte, risultare frammentarie e poco chiare.

\section{Analisi dei Dati \\ Gli atti con valore affettivo}

Il primo esempio illustra la tipologia di atti detti "con valore affettivo". La sequenza, prodotta da due studentesse, Luciana $(\mathrm{L})$ e Flora $(\mathrm{F})^{3}$, è stata raccolta durante una conversazione in cui stavano esprimendo apprezzamenti sulla città di Rio.

\section{Esempio $1^{4}$}

$1 \mathrm{~L}$ : è molto bello il pão de açúcar il bonde il bondinho

2 F: è molto bello

Luciana parla del "pão de açucar", una località turistica di Rio, con un accento marcatamente brasiliano, poi passa al cambio di codice, optando per il termine "bonde", noto simbolo turistico di Rio, il quale è preferito a "tram" per la carica espressiva che ha. Come si può notare, il cambio di codice non nasce dal bisogno di colmare un vuoto linguistico, ma è usato per rafforzare il senso di appartenenza ad una certa comunità.

Il fatto che tali produzioni non siano seguite da interventi riparativi ${ }^{5}$ conferma che può trattarsi di una pratica legittimata da entrambi gli interlocutori i quali, per il fatto di condividere la stessa LM, attingono a questa per parlare di una dimensione a loro nota che, se espressa

3 Per preservare l'anonimato i nomi degli studenti sono fittizi.

4 Norme per la trascrizione: Ad, An, F, L, M, Pa, C: iniziali utilizzate per indicare gli alunni; P: iniziale usata per indicare l'insegnante;,..,$\ldots$, ecc.: pausa breve; =: due turni di parole in sovrapposizione; ((inc)), ((risate)): la parentesi doppia indica una parte della conversazione incomprensibile o dei commenti sulla trascrizione, come risate ecc.; -: indica un'interruzione brusca; :::: i due punti ripetuti per tre volte di seguito indicano un suono prolungato; ?: intonazione ascendente; !: intonazione discendente;

testo: fenomeno che si desidera mettere in risalto.

5 Con questo termine coniato da Schegloff, Jefferson, Sacks (1977), si intende il trattamento dei problemi che possono sorgere durante l'uso linguistico interattivo (Van Lier, 1988). La nozione di riparazione è molto più ampia rispetto $a$ quella di correzione, poiché presuppone il continuo lavoro di negoziazione che ha luogo fra gli interattanti. Le riparazioni sono classificate da Schegloff, Jefferson, Sacks (1977) in riparazioni autoiniziate, in cui l'errore è correto dal parlante stesso che lo commette, e in riparazioni eteroiniziate, eseguite dall'ascoltatore 
diversamente, soffrirebbe, forse, di un impoverimento a livello affettivo ed espressivo.

Nella sequenza successiva agli studenti è stato chiesto di immaginare di dover organizzare un pranzo a casa loro. Nel corso della conversazione Caterina (C) realizza un cambio di codice dall'italiano al portoghese-brasiliano quando parla del "miojo" (riga 11), una pietanza a preparazione istantanea molto in voga fra gli studenti. In questo modo, attraverso il passaggio dalla LS alla LM, sottolinea la propria appartenenza alla cultura brasiliana, condivisa dal resto dei membri del gruppo.

\section{Esempio 2}

$3 \mathrm{C}$ : po- possiamo fare anche la pasta?

$4 \mathrm{~L}$ : spaghetti quali? penne

5 Pa: penne ((risate)) va bene

$6 \mathrm{C}$ : riso e fagioli è più facile ((ride))

7 L: no::: le penne molto più facile che riso e fagioli le penne metti l'acqua e si bolle

9 C: e il sugo come si fa?

$10 \mathrm{~L}$ : lo compriamo pronto ((risate))

$11 \mathrm{C}$ : ma è più facile del miojo?

12 L: è sì

$13 \mathrm{~Pa}$ : è la salsa di pomodoro è già tutta pronta

14 L: mettiamo un po' di:::

15 Pa: aggiungi un po' d'acqua

16 L: un po' d'acqua un po' di::: cipolle e noce moscata va bene?

$17 \mathrm{C}$ : mi piace il sugo bianco

\section{Gli atti di potere}

Un'altra tipologia appartiene alla categoria denominata "atti di potere". Si tratta di atti usati dai parlanti più esperti per rivendicare una certa superiorità rispetto agli altri membri del gruppo e si manifesta attraverso una serie di attività di riparazione che possono evolversi in sequenze laterali di carattere pedagogico (ORLETTI, 2000, p. 121). Le sequenze riparative innescano un processo di negoziazione del significato, in base al quale i parlanti utilizzano le risorse che hanno a loro disposizione per raggiungere, dopo successivi aggiustamenti, la comprensione e la formulazione di un messaggio congiuntamente elaborato. A loro volta determinano la 
riconfigurazione del quadro partecipativo attraverso dei momenti in cui l'attenzione è tutta concentrata sulla lingua.

Si veda l'esempio 3 , in cui, sebbene si tratti di una conversazione tra pari, in termini quantitativi lo spazio interazionale a disposizione di Luciana è inferiore se paragonato a quello di Maria, anche se avere più spazio interazionale è piuttosto indice di debolezza che di potere (ORLETTI, 2000, p. 14). Sul piano linguistico è possibile notare fin dal primo turno una disparità fra la competenza di Maria (M) e quella di Luciana. Si veda, ad esempio, il frequente uso di riformulazioni (riga 20), esitazioni (riga 18), prolungamenti del suono (riga 18) e richieste dirette (riga 20), rivolte all'interlocutore che possiede una maggiore competenza linguistica. In virtù del fatto che Luciana è molto più abile ed esperta linguisticamente, l'interazione tra i due parlanti dà vita ad una serie di interventi riparativi, attraverso i quali, per soddisfare le richieste d'aiuto di Maria ${ }^{6}$, Luciana modifica il proprio input e rettifica le infrazioni commesse da Maria tramite una modalità poco "esposta" (JEFFERSON, 1983), cioè poco evidente, tanto che nell'ultimo turno, quello in cui la dominanza strategica dell'“esperto linguistico" raggiunge il proprio culmine (riga 26), Luciana oltre ad usare un tono di voce più basso, per evitare di interrompere il flusso tematico, opta per il passaggio al portoghese-brasiliano. Nonostante i ruoli di "docente" e di "apprendente", ovvero di "esperto" e di "non esperto", che si profilano all'interno della sequenza pedagogica, grazie anche all'attività di gruppo che determina in un certo senso uno status a priori egualitario, riducendo drasticamente l'asimmetria tipica delle interazioni tra insegnante ed allievi (cfr. NUSSBAUM e UNAMUNO, 2000; CAMBRA, 2003, 2005), gli interattanti non incorrono in problemi di "faccia" (BROWN e LEVINSON, 1987)"

\section{Esempio 3}

$18 \mathrm{M}$ : penso che sia::: eh::: il prime::: il nome delle ragazze

19 L: di queste qua

$20 \mathrm{M}$ : sì cacridobo ((leggendo)) ognuna::: il nome di ognuna comincia con queste lettere io penso che sia un diario scritto a quattro mani::: penso che sia un diario::: un documentario::: come si dice in italiano?

$21 \mathrm{~L}:$ un documentario

$22 \mathrm{M}$ : un documentario sull'era fascista

$23 \mathrm{M}$ : non ho capito quella quel 1936 era guerra o no?

6 Gli interventi riparativi sono molto più ricorrenti nelle attività in gruppo poiché gli apprendenti non sono soggetti ad una possibile valutazione da parte dell'insegnante (CAMBRA, 2005).

7 Come ha mostrato magistralmente GOFFMAN (1967), gli interattanti utilizzano delle procedure specifiche per salvaguardare la propria e l'altrui faccia da possibili minacce. Tutte le interazioni, soprattutto quelle pubbliche, possono potenzialmente costituire un conflitto per le facce dei partecipanti. Nella classe di lingua, a causa dell'asimmetria linguistica, le continue valutazioni e correzioni fatte dall'insegnante, possono essere vissute dagli apprendenti come minacce (cfr. CAMBRA, 2003). 
24 L: no

$25 \mathrm{M}$ : ma c'era una guerra?

26 L. sì sì più che una guerra::: una corsa::: direi in portoghese ((a voce bassa)) eu pego você ((inc))

$27 \mathrm{M}$ : obrigado

I parlanti possono ricorrere oltre che alla loro LM anche ad altre lingue che compongono il loro repertorio linguistico ${ }^{8}$ per rivendicare una certa superiorità sugli altri membri del gruppo. Nella sequenza 4 vediamo come da un'attività, che consiste nell'analizzare i tempi verbali contenuti in un depliant turistico, prende vita una vera e propria discussione tra gli studenti e l'insegnante che sta passando fra il gruppo per accertarsi che il compito assegnato venga svolto correttamente. Nel corso dello scambio dialogico gli studenti per prima cosa infrangono il sistema della presa del turno preferendo l'autoselezione all'eteroselezione' ${ }^{9}$ La sequenza si apre con una richiesta di Paolo $(\mathrm{Pa})$ che provoca una sospensione dell'attività in corso, indirizzata a colui che detiene una maggiore autorità e competenza in materia di lingua, ovvero l'insegnante (cfr. ORLETTI, 2000; FELE; PAOLETTI, 2003; CAMBRA, 2003). L'insegnante che non capisce la domanda che le è stata rivolta (riga 28), in pratica gli studenti vogliono sapere se è possibile esprimere il futuro con delle forme perifrastiche ${ }^{10}$, prima prende tempo (righe 29 e 32), poi prova a fornire delle risposte che però non sono esaurienti (righe $41 \mathrm{e} 46$ ). A questo punto Adriana (Ad) ostenta la sua competenza in altre lingue nei confronti dell'insegnante, citando degli esempi in inglese (righe 33 e 43). L'insegnante allora riformula la risposta data nel turno precedente (riga 46) che viene contesta da Paolo tramite un atto che destabilizza l'autorità del professore (riga 47). Infine, per salvarsi la faccia e riaffermare il suo ruolo, l'insegnante si riappropria del turno di parola e termina la sequenza richiamando gli studenti ai loro doveri "facciamo una cosa è::: sono curiosa anche io di sapere la risposta perché non me la ricordo realmente ma ne parliamo dopo".

8 Per repertorio intendiamo la totalità di forme linguistiche, regolarmente utilizzate nel corso di interazioni sociali significative (GUMPERZ, 1964).

9 Negli incontri 'orchestrati', a differenza della conversazione ordinaria, solo uno dei partecipanti ha il diritto esclusivo di determinare quando gli altri partecipanti possono parlare (FELE e PAOLETTI, 2002).

10 Forme influenzate dall'inglese che si stanno espandendo nell'italiano standard (BERRETTA, 1993). 


\section{Esempio 4}

28 Pa: ((rivolto all'insegnante)) è una forma di parlare al passato una cosa che::: è stata già $29 \mathrm{P}: \mathrm{mmm}$ che è già successa

30 Pa: sì

31 Ad: però::: si estende anche al::: al presente

32 P: va bene allora si il vostro dubbio io non l'ho ancora capito ((sorride))

33 Ad: tipo $i$ have studying

34 P: ho studiato

35 Ad: ho studiato

36 P: l'idea di::: aver appena finito un'azione

37 Pa:è!

38 Ad: già fatto

39 P non è un modo verbale

40 Ad:è già fatto

$41 \mathrm{P}$ : sì allora il fatto è che ma la::: ((inc)) nel passato ma è finita ho studiato italiano per tre anni io ho finito

42 Pa: è una che non è finita ha è iniziata

43 Ad: è come:::: $i$ be doing

$44 \mathrm{~Pa}$ : è come questo c'è?

45 Ad: io ho studiato

46 P: ho studiato italiano per tre anni

47 Pa: però:::: non è finita!

48 Ad: io sto studiando ma io ho già cominciato a studiare tre anni fa non c'è come in inglese?

$49 \mathrm{P}$ : fatemi pensare adesso non mi viene in mente facciamo una cosa è::: sono curiosa anche io di sapere la risposta perché non me la ricordo realmente ma ne parliamo dopo

Nel caso appena analizzato lo studente che ricorre all'inglese lo fa per dimostrare di saperne di più del proprio insegnante. Gli atti di potere quindi danno luogo a una serie di attività di negoziazione di natura diversa. Se nell'esempio 3 il parlante più esperto mette a disposizione la propria dominanza strategica e linguistica in un' atmosfera collaborativa e interviene per facilitare i dubbi lessicali attraverso degli atti in LM che non producono né brusche interruzioni rispetto al proseguimento dell'attività in corso, né minacciano la faccia dell'altro e la cui funzione 
principale è quella di aiutare colui che si trova in difficoltà, nell'esempio 4 la lingua inglese e la LM sono usate dagli studenti per ostentare le proprie competenze rispetto all'altro, in questo caso l'insegnante. Questa tipologia di atti che determinano una sospensione temporanea dell'attività in corso e la riconfigurazione del quadro partecipativo (GOFFMAN, 1987) si presenta come fenomeno altamente complesso, a causa della momentanea ridefinizione dei rispettivi ruoli che coinvolge la messa in gioco dell'immagine personale, la "faccia" (cfr. BROWN e LEVINSON, 1987).

\section{Gli atti di collaborazione e gli atti di difficoltà}

Nel corpus abbiamo individuato la presenza di una serie di sequenze in cui, a causa della prossimità fra le lingue e per il fatto che gli studenti condividono la stessa LM, prendono vita delle neoformazioni che, per la loro singolarità, serviranno per conferire alla conversazione toni scherzosi. Nel frammento che segue troviamo un esempio di atto di collaborazione. È evidente il tono scherzoso e l'atteggiamento complice dei due studenti, Paolo (Pa) e Caterina (C), mentre stanno rispondendo ad alcune domande relative a una sequenza video. La presenza di interventi riparativi auto ed eteroiniziati, tipica della conversazione fra pari, permette agli apprendenti di non perdere la faccia, come invece accade quando a correggere l'errore è l'insegnante (si veda la riga 54 in cui Paolo manifesta il proprio dubbio fra "cartello", termine corretto, e "cartace", stranierismo composto dal sostantivo portoghese "cartaz", più il suffisso italiano poco produttivo "ace").

La sequenza si conclude con la ripresa (riga 56) da parte di Paolo della neoformazione, questa volta però non si tratta più di una creazione interlinguistica usata per colmare un dubbio linguistico, visto che è già stato dissipato nel turno precedente da Caterina (riga 55), ma ha più che mai un valore ironico, poiché è impiegato intenzionalmente da Paolo per allentare la tensione e creare un clima piacevole. In questo modo Paolo rivela la sua capacità di saper giocare con una nuova lingua che ha molto in comune con la lingua di partenza, tanto da suscitare le risate della compagna. 


\section{Esempio 5}

50 Pa: ((leggendo)) quali elementi ti hanno permesso di dedurre il contesto

$51 \mathrm{C}$ : le da...la date?

$52 \mathrm{~Pa}$ : le date

$53 \mathrm{C}$ : le date c'era un cartello scritto l'etiopia

54 Pa: sì cartello o cartace come si dice cartello o cartace penso che cart- non so

$55 \mathrm{C}$ : cartello

56 Pa: cartace no? é muito bom ((risate))

Essendo la classe di lingua un luogo sociale singolare, dove il bilinguismo ${ }^{11} \mathrm{è} \mathrm{in} \mathrm{fase} \mathrm{di}$ costruzione e dove gli interattanti dispongono di competenze molto asimmetriche fra la LM e la lingua target (PY, 1994), in presenza di attività linguisticamente complesse, possono verificarsi degli atti da noi denominati di "contrasto-difficoltà" in cui gli studenti manifestano la propria incompetenza linguistica nei confronti dell'attività che stanno svolgendo. Nell'esempio 6, presentiamo un frammento in cui i vari gruppi esprimono dei consigli attraverso l'impiego del condizionale presente (dovresti dire al tuo vicino). Questo esercizio presenta una doppia difficoltà: da una parte Caterina (C) e Flora (F) non possiedono il lessico necessario per riferirsi alle situazioni proposte, dall'altra devono trasformare le loro opinioni in testo scritto. Nonostante, come è già stato sottolineato, il lavoro a coppie o in piccoli gruppi determini uno status a priori egualitario fa gli allievi i quali si sentono maggiormente a loro agio dato che non sono soggetti ad una valutazione da parte dell'insegnante (NUSSBAUM; UNAMUNO, 2000; CAMBRA, 2003; 2005), è possibile notare che Caterina, dopo aver rivolto a Flora una richiesta diretta d'aiuto (riga 58), forse anche a causa del registratore che in un certo senso sostituisce la presenza dell'insegnante, giustifica, dopo aver ottenuto la risposta, la propria incompetenza in LS, attraverso un atto di difficoltà in LM (riga 60).

\section{Esempio 6}

57 C: uscire la sera gli gli piace uscire la sera praticare gli sport e::: ((legge)) dovrebbe cercare un appartamento vicino al suo lavoro perché di questo modo potrai avere più tempo di praticare gli sport e anche

$58 \mathrm{~F}:$ e anche?

59 C: e também

60 F: sì e anche essas palavrinhas de ligações são as piores eu não consigo pensar de acordo com que eu sei eu penso do jeito que eu sou em português ((ridono))

11 Concordiamo con MACKEY (1976) e GROSJEAN (1982), quando affermano che il bilinguismo è un fenomeno individuale, presente nella maggior parte della popolazione del pianeta. 


\section{Conclusioni}

Attraverso questo lavoro, abbiamo identificato alcuni atti di identità espressi da apprendenti di italiano LS durante lo svolgimento di attività di gruppo.

Gli "atti con valore affettivo" sono usati dagli studenti per riferirsi ad un aspetto che appartiene al proprio paese ed è condiviso dai compagni. Visto che in alcuni casi le diverse identità che compongono ciascun individuo possono, a seconda delle circostanze, combinarsi fra loro (si vedano, ad esempio, RAMPTON, 1995; ANTAKI; WIDDICOMBE, 1998; DE FINA, 2007; DE FINA, SCHIFFRIN e BAMBERG, 2006; CILIBERTI, 2007), possiamo dire che, nei nostri dati, attraverso gli atti con valore affettivo, l'individuo permette all'identità di parlante di una determinata LM di prevalere su quella di studente di una determinata LS. Potremmo anche pensare a una sorta di allineamento con il macro ambiente università o società, a scapito del micro ambiente classe.

Gli "atti di potere" sono usati dagli apprendenti per dimostrare una certa superiorità sui compagni e dipendono sia dalla sensibilità e dalla consapevolezza metalinguistica che il discente ha maturato in LS, sia dalle conoscenze del mondo che ha acquisito nel corso della propria esperienza. Gli studenti possono usare la loro LM per mostrarsi dei bravi studenti e aiutare colui che si trova in difficoltà per mezzo di una serie di sequenze riparative che non pregiudicano la "faccia" dell'altro, oppure possono ricorrere, oltre che alla loro LM, anche ad altre lingue che compongono il loro repertorio linguistico per cercare di imporsi sul gruppo e raggiungere o mantenere la figura del leader. In questo modo, lo studente compie un vero e proprio atto di potere che gli consente di assumere una posizione di dominio non solo sui compagni, ma anche sull'insegnante.

Abbiamo notato che lo studente di una LS affine alla propria LM sembra propenso a affrontare dei rischi e a cercare di dare una risposta anche se linguisticamente sbagliata, invece di scegliere il silenzio o di intervenire solo nel caso in cui si senta sicuro. Per esprimere gli "atti di collaborazione", gli apprendenti usano delle neoformazioni in cui alla parola in LM vengono aggiunti suffissi tipici della lingua italiana. Questo procedimento si verifica in modo collaborativo, in un'atmosfera di complicità tra gli studenti che dimostrano di voler arrivare a un accordo. In presenza di attività linguisticamente complesse, possono prendere vita degli atti da noi denominati "di contrasto-difficoltà", in cui gli studenti manifestano in LM la propria mancanza di competenza linguistica nei confronti dell'attività che stanno svolgendo, considerata particolarmente difficile. Lo studente si oppone in questo modo allo svolgimento del compito. 
Per concludere, riteniamo che l'osservazione e l'analisi critica di quello che gli apprendenti fanno e dicono all'interno della classe di lingua possano costituire un importante strumento ai fini della formazione iniziale e continua degli insegnanti, dato che consentono di riflettere sul

modo in cui i protagonisti della scena educativa si relazionano fra loro di fronte a nuovi saperi.

\section{Riferimenti bibliografici}

ANTAKI, C.; WIDDICOMBE, S. Identities in talk. London: Sage, 1998.

BERRETTA, M. Morfologia. In SOBRERO, A.A. (Org.). Introduzione all'italiano contemporaneo. Le strutture. Bari: Laterza, 1993, pp. 193-245.

BLOCK, D. Second language identities. Continuum: London, 2007.

BROWN, P.; LEVINSON, S. Politeness: some universals in language usage. Cambridge: Cambridge University Press, 1987.

CAMBRA, M. L'(auto-)observation et l'analyse de l'interaction em sous-groupes par de futurs enseignants de FLE: l'evolution des représentations. In Le Français dans le Monde, Recherches et Applications: les interactions en classe de langue, numéro spécial: 148-159, juillet, 2005.

. Une approche ethnographique de la classe de langue. Paris: Didier, 2003.

CELADA, M.T. Frontera discursiva. Desencuentro de sentidos y alteridade. In Páginas de Guarda, n. 6: 40-55, 2008.

CILIBERTI, A. La costruzione interazionale di identità: repertori linguistici e pratiche discorsive degli italiani in Australia. Pavia: Franco Angeli, 2007.

CLARK, H. H.; CLARK, E. V. Psychology and language. New York: Harcourt Brace Jovanovich, 1977.

CONSIGLIO D'EUROPA. Quadro Comune Europeo di Riferimento per le Lingue: apprendimento insegnamento valutazione. Firenze: La Nuova Italia-Oxford, 2002.

DE FINA, A. Code-switching and the construction of ethnic identity in a community of practice. In Language in Society, v. 36, n. 3: 371-392, 2007.

DE FINA, A.; SCHIFFRIN, D.; BAMBERG, M. Discourse and identity. Cambridge: Cambridge University Press, 2006.

DURANTI, A. Linguistic anthropology. Cambridge: Cambridge University Press, 1997. 274 p.

ELLWOOD, C. Undoing the knots: identity transformations in a study abroad programme. In Educational Philosophy and Theory, v. 43, n. 9: 960-978, 2011.

Questions of classroom identity: what can be learned from codeswitching in classroom 
peer group talk? In Modern Language Journal, v. 92, n. 4: 538-557, 2008.

FELE, G.; PAOLETTI, I. L'interazione in classe. Bologna: Il Mulino, 2003.

FIRTH, A.; WAGNER, J. On discourse, communication, and some fundamental concepts in SLA research. In Modern Language Journal, v. 81, n. 3: 285-300, 1997.

GAJO, L.; KOCH, P.; MONDADA, L. La pluralité des contextes et des langues: une approche interactionnelle de l'acquisition. In Bulletin Suisse de Linguistique Appliqué, v. 64, n. 1: 61-86, 1996.

GOFFMAN, E. Forme del parlare. Bologna: Il Mulino, 1987. Il rituale dell'interazione. Bologna: Il Mulino, 1967.

GROSJEAN, P. Life with two languages: an introduction to bilingualism. Cambridge: Harvard University Press, 1982.

GUMPERZ, J. Linguistic and social interaction in two communities. In American Anthropologist, v. 66, n.6: 137-153, 1964.

JEFFERSON, G. On exposed and embedded correction in conversation. In Studium Linguistik, v. 14, n. 1: 58-68, 1983.

JOHNSON, K.; GOLOMBEK, P. Teachers' narrative inquiry as professional development. Cambridge: Cambridge University Press, 2002.

KRAMSCH, C. The multilingual subject. Oxford: Oxford University Press, 2007.

LANTOLF, J.; GENUNG, P. "I'd rather switch than fight": an activity theoretic study of power, success, and failure in a foreign language classroom. In KRAMSCH, C. (Org.). Language acquisition and language socialization. London: Continuum, 2002, pp. 175-196.

LE PAGE, R.B.; TABOURET-KELLER, A. Acts of identity: creol-based approaches to language and ethnicity. Cambridge: Cambridge University Press, 1985.

LIDDICOAT, A.; CROZET, C. Acquiring French interactional norms through instruction. In ROSE, K.; KASPER, G. (Orgs.). Pragmatics in language teaching. Cambridge: Cambridge University Press. 2001.pp. 125-144.

MACKEY, W.F. Bilinguisme et contact de langues. Paris: Klincksieck, 1976.

NORTON, B. Language and identity. In TESOL Quarterly, v. 31, n. 3 (special issue): 1997. . Identity and language learning. London: Longman, 2000.

NORTON PEIRCE, B. Social identity, investment, and language learning. In TESOL Quarterly, v. 29, n. 1: 9-31, 1995.

NUNAN, D. Designing tasks for the communicative classroom. Cambridge: Cambridge University Press, 1989. 
NUNAN, D.; CHOI, J. Language and culture: reflective narratives and emergence of identity. Cambridge: Cambridge University Press, 2010.

NUSSBAUM, L.; UNAMUNO, V. Fluidité et complexité dans la construction du discourse entre apprenants de langues étrangères. In AILE, v. 12, n. 1: 22-50, 2000.

ORLETTI F. La conversazione diseguale: potere e interazione. Roma: Carocci, 2000.

PAVLENKO, A. Language learning memoirs as gendered genre. In Applied Linguistics, v. 22, n. 2 : 213-240, 2001.

PAVLENKO, A.; BLACKLEDGE, A. Negotiation of identities in multilingual setting. Clevedon: Multilingual Matters, 2004.

PEKAREK DOEHLER, S. Conceptual changes and methodological challenges: on language and learning from a conversation analytic perspective on SLA. In P. SEEDHOUSE, P.; WALSH, S.; JENKS, C. (Orgs.). Conceptualising Learning in Applied Linguistics. Basingstoke, UK: Palgrave Macmillan. 2010, pp. 105-127.

. CA for SLA: analyse conversationnelle et recherché sur l'acquisition des langues. In Revue Française de Linguistique Appliquée, n. special: 123-137, 2006.

PY, B. Place des approches interactionnistes dans l'étude des situations de contacts et d'acquisition. In VERONIQUE, D. (Org.). Créolisation et acquisition des langues. Aix en Provence: Publications des Universités de Provence. 1994, pp. 136-147.

RAMPTON, B. Crossing: language and ethnicity among adolescent. New York: Longman, 1995.

SERRANI-INFANTE, S. Identidade e segundas línguas: as identificações no discurso. In: SIGNORINI, I. (Org.) Língua (gem) e identidade. Campinas: Mercado das Letras, 1997. pp. 231-264.

SCHEGLOFF, E.; JEFFERSON, J.; SACKS, H. The preference for self-correction in the organization of repair in conversation. In Language, v. 53, n. 2: 361-382, 1977.

STERN, H. Fundamental concepts of language teaching. London: Oxford University Press, 1983.

VAN LIER, L. The classroom and the language learner. London: Longman, 1988.

WATSON-GEGEO, K. Classroom ethnography. In HORNBERGER, N.H.; CORSON, D., (Orgs.). Encyclopedia of language and education, v. 8, Research Methods in Language and Education, The Netherlands, 1997, pp. 135-144. 